\title{
Escore TIMI no Infarto Agudo do Miocárdio Conforme Níveis de Estratificação de Prognóstico
}

\author{
TIMI Risk Score for Acute Myocardial Infarction According to Prognostic Stratification
}

\section{Jaqueline Locks Pereira1, Thiago Mamôru Sakae1, Michele Cardoso Machado¹, Charles Martins de Castro1,2}

Universidade do Sul de Santa Catarina'; Hospital Nossa Senhora da Conceição Florianópolis², SC - Brasil

\section{Resumo}

Fundamento: O escore de risco TIMI (thrombolysis in myocardial infarction) é derivado de ensaio clínico envolvendo pacientes elegíveis para fibrinólise. Como o perfil de risco desses casos difere do encontrado em populações não selecionadas, é importante que se analise a aplicabilidade do escore em condições clínicas habituais.

Objetivo: Avaliar o manejo e a evolução hospitalar de pacientes internados com infarto agudo do miocárdio conforme estratificação de risco pelo escore TIMI.

Métodos: Foram avaliados, retrospectivamente, 103 casos de infarto agudo do miocárdio com supradesnivelamento do segmento ST, admitidos no Hospital Nossa Senhora da Conceição, em Tubarão, nos anos de 2004 e 2005. Os casos foram analisados em três grupos de risco de acordo com o escore TIMI.

Resultados: A mortalidade hospitalar pós-infarto foi de 17,5\%. No grupo de baixo risco não houve óbito. A mortalidade foi de $8,1 \%$ no grupo de médio risco e de $55,6 \%$ no de alto risco. $O$ risco de morte para casos de alto risco foi 14,1 vezes maior em relação aos casos de médio e baixo risco (IC95\% $=4,4$ a 44,1 e $p<0,001)$. A chance de receber fibrinolítico foi $50 \%$ menor no grupo de alto risco em relação ao de baixo risco $($ IC95\%= $0,27-0,85 ; p=0,004)$.

Conclusão: Houve um aumento progressivo na mortalidade e na ocorrência de complicações hospitalares conforme estratificação pelo escore TIMI. Pacientes de alto risco receberam trombolítico menos frequentemente que pacientes de baixo risco. (Arq Bras Cardiol 2009; 93(2) : 105-112)

Palavras-chave: Prognóstico clínico dinâmico, infarto do miocárdio, mortalidade intra-hospitalar.

\section{Summary}

Background: The TIMI (Thrombolysis in Myocardial Infarction) risk score is derived from clinical trial involving patients who are eligible for fibrinolysis. As the risk profiles of these cases differ from those found in non-selected populations, it is important to review the applicability of the score in usual clinical conditions.

Objectives: To evaluate the management and clinical evolution of hospital inpatients with acute myocardial infarction, according to risk stratification by the TIMI score.

Methods: We evaluated, retrospectively, 103 cases of acute myocardial infarction with ST-segment elevation admitted to the Hospital Nossa Senhora da Conceição - Tubarão, in 2004 and 2005. The cases were analyzed in three risk groups according to the TIMI score.

Results: The hospital mortality after infarction was $17.5 \%$. In the low-risk group there was no death. The mortality was $8.1 \%$ in the medium risk group and $55.6 \%$ in the high-risk group. The risk of death in cases of high risk was 14.1 times higher than in the cases of medium and low risk $(95 \% \mathrm{Cl}=4.4$ to 44.1 and $p<0.001)$. The chance of receiving fibrinolytic was $50 \%$ lower in the high-risk group in relation to the low risk group $(95 \% \mathrm{Cl}=0.27$ to $0.85, \mathrm{p}=0.004)$.

Conclusion: There was a progressive increase in mortality and incidence of in-hospital complications according to the stratification by the TIMI score. High risk patients received thrombolytic less frequently than the patients at low risk. (Arq Bras Cardiol 2009; 93(2) : 100-106)

Key Words: Clinical - dynamic prognosis; myocardial infarction; hospital mortality.

Full texts in English - http://www.arquivosonline.com.br 


\section{Introdução}

O infarto agudo do miocárdio (IAM) constitui um dos maiores problemas de saúde pública nos países industrializados ${ }^{1}$. Nos Estados Unidos, a doença coronariana é responsável por 53\% das mortes por doença cardiovascular, ocorrendo cerca de 780 mil casos de infarto por ano em americanos com idade acima de 65 anos $^{2}$. Embora não se saiba o número de infartos que ocorrem anualmente no Brasil, estima-se em 300 a 400 mil, tornando o IAM a principal causa isolada de morte no país ${ }^{1,3}$.

Nas últimas décadas, o surgimento das unidades coronarianas, o uso disseminado de fibrinolítico, betabloqueadores, ácido acetilsalicílico e o desenvolvimento da angioplastia transluminal coronariana, contribuíram para a redução da taxa de mortalidade hospitalar pós-IAM de 30\% na década de 50, para os atuais $6 \%$ a $10 \%{ }^{3-5}$. Entretanto, a variabilidade na mortalidade hospitalar é considerável e está relacionada a diferenças nos perfis de gravidade e na qualidade da assistência médica prestada ${ }^{3-6}$.

Nesse contexto, a utilização de escores prognósticos para estratificação de risco pós-infarto torna-se uma ferramenta importante no manejo desses pacientes ${ }^{3}$. A determinação do prognóstico do paciente possibilita a indicação precoce de procedimentos complexos como cinecoronariografia, angioplastia e revascularização cirúrgica para casos de alto risco e tempo menor de internação para indivíduos de baixo $\operatorname{risco}^{7,8}$.

O escore $\mathrm{TIMI}^{9}$, para infarto agudo do miocárdio com supradesnivelamento do segmento ST, foi descrito originalmente baseado em oito variáveis ${ }^{9}$. Esse escore tem sido apontado como um método simples, facilmente aplicável à beira do leito e com bom poder discriminatório em relação às complicações clínicas e mortalidade precoce ${ }^{9,10}$.

Pesquisadores têm enfatizado a necessidade de se testar a aplicabilidade clínica de escores de risco em populações diferentes do estudo original ${ }^{11}$. Isso porque os critérios de inclusão e exclusão de cada estudo podem selecionar perfis de risco diferentes, que exercem influência nos fatores prognósticos retidos no escore final e na validade clínica em populações diferentes da original ${ }^{11,12}$.

Considerando todos esses aspectos, a aplicação do escore TIMI permite avaliar o desempenho do mesmo em predizer o risco de morte e de complicações pós-infarto em condições clínicas habituais. Além disso, possibilita identificar diferenças no manejo dos pacientes conforme o prognóstico determinado pelo escore.

\section{Métodos}

Um estudo observacional longitudinal, retrospectivo, de 103 pacientes internados no Hospital Nossa Senhora da Conceição, em Tubarão, SC; no período de 01/01/2004 a 31/12/2005, com diagnóstico de infarto agudo do miocárdio com supradesnivelamento do segmento ST. A coleta de dados foi realizada através de consulta ao prontuário médico e preenchimento de protocolo elaborado pelos pesquisadores.Os critérios utilizados para o diagnóstico de Infarto Agudo do Miocárdio com supradesnivelamento de ST foram os seguintes: presença de dor torácica ou outra sintomatologia sugestiva de IAM; elevação do segmento ST na admissão ou durante evolução hospitalar em duas ou mais derivações contíguas - sendo maior que 0,2 $\mathrm{mV}$ nas derivações $\mathrm{V} 1, \mathrm{~V} 2$ ou $\mathrm{V} 3$ e maior que $0,1 \mathrm{mV}$ nas outras derivações; bloqueio de ramo esquerdo novo ou presumivelmente novo; e curva típica da enzima creatinofosfoquinase total e sua fração $\mathrm{MB}^{3}$.

Os pacientes foram divididos em grupos de risco conforme a pontuação no escore TIMI (anexo 1). A pontuação mínima pelo escore TIMI é zero, e a máxima 14. Todos os 103 casos receberam uma pontuação entre 0 e 13. Isso por que a variável peso não estava registrada nos prontuários. Considerou-se, dessa forma, baixo risco pontuação entre 0 e 2; médio risco pontuação de 3 a 5; e alto risco pontuação maior que $5^{13}$.

As variáveis estudadas foram: idade, gênero, procedência, tempo de internação, frequência cardíaca, pressão arterial sistêmica, parada cardiorrespiratória à admissão, tempo entre dor e primeiro atendimento, classe de Killip-Kimball à admissão, localização eletrocardiográfica do infarto, valor máximo atingido pelas enzimas cardíacas e complicações e uso de trombolítico.

Foram classificados como diabéticos aqueles que, em exames prévios ou durante a internação, apresentaram níveis de glicemia de jejum compatíveis com o diagnóstico. Hipertensão arterial e angina foram consideradas naqueles com diagnóstico prévio ao IAM.

O principal desfecho analisado foi a mortalidade hospitalar pós-infarto. Em relação às complicações durante a internação hospitalar, considerou-se: taquicardia ventricular sustentada, fibrilação ventricular, assistolia, e bloqueio atrioventricular de alto grau, os quais foram considerados positivos quando registrados em prontuário ou quando presentes em algum dos eletrocardiogramas realizados durante a hospitalização. $\mathrm{O}$ diagnóstico de choque cardiogênico levou em consideração a necessidade de drogas vasoativas e/ou registro do mesmo em prontuário. A dor precordial recorrente foi levada em conta apenas se anotada em prontuário médico. Foram considerados Killip II os casos com estertores basais ou 3a bulha, e Killip III os casos com estertores audíveis até o campo médio33.

A análise dos dados foi feita através do programa SPSS $®$ 8.0, e os resultados apresentados como frequências ou médias com o desvio padrão associado. Os testes do qui-quadrado, ou exato de Fisher, quando apropriado, foram usados para testar a significância estatística de diferenças observadas nas proporções das variáveis categóricas estudadas, considerandose estatisticamente significativo um valor de $\mathrm{p}<0,05$ bi-caudal. Foram calculados intervalos de confiança de 95\% (IC95\%) para os riscos relativos (RR) estimados na amostra.

O estudo foi aprovado pelo Comitê de Ética e Pesquisa da Universidade do Sul de Santa Catarina - UNISUL.

\section{Resultados}

A média de idade encontrada foi de $60 \pm 12$ anos, sendo de $58,3 \pm 10,9$ anos para o sexo masculino e 63,6 \pm 13,5 anos para o sexo feminino. Quanto à procedência, o município de origem foi Tubarão, em 39,8\% dos casos. 


\section{Artigo Original}

O tempo médio entre o início dos sintomas e o primeiro atendimento médico foi de $6,7 \pm 8,6$ horas. Para os pacientes de Tubarão, esse tempo foi de 4,5 $\pm 4,6$ horas, enquanto para os de municípios vizinhos foi de 8,2 $\pm 10,2$ horas. O tempo médio entre o início do quadro e a procura por assistência médica foi de $6,8 \pm 10$ horas para o sexo masculino e de 6,5 \pm 4,8 horas para o feminino.

A localização do infarto apontou para um acometimento mais frequente da parede inferior $(51,5 \%$ dos casos) e para uma maior mortalidade quando o infarto afetou a parede anterior extensa (33,3\% dos casos). Não houve nenhum caso de bloqueio de ramo esquerdo (Tabela 1).

A mortalidade hospitalar pós-IAM foi de 17,5\%. Do total de óbitos, $66,7 \%$ ocorreram nas primeiras 48 horas. Houve um aumento progressivo na mortalidade conforme aumentou o risco determinado pelo escore TIMI. O risco de morte no grupo de alto risco, comparado ao de médio e baixo risco, foi quase 14,1 vezes maior (IC95\%: 4,4 - 44,9; $\mathrm{p}<0,001$ ) (Tabela 2).

$\mathrm{Na}$ amostra, 58,3\% dos pacientes receberam trombolítico, e nesse grupo, a mortalidade foi de $10 \%$. Entre os pacientes

Tabela 1 - Perfil dos pacientes e mortalidade intra-hospitalar pós-infarto agudo do miocárdio. Tubarão-SC, 2004 a 2005

\begin{tabular}{|c|c|c|}
\hline Variáveis & $\mathrm{n}^{\circ} /(\%)$ & Mortalidade (\%) \\
\hline \multicolumn{3}{|l|}{ Características demográficas } \\
\hline Sexo masculino & $68(66,0)$ & 10,3 \\
\hline Sexo feminino & $35(34,0)$ & 31,4 \\
\hline Idade entre 18 e 74 anos & $89(86,4)$ & 9,0 \\
\hline Idade igual ou superior a 75 anos & $14(13,6)$ & 71,4 \\
\hline \multicolumn{3}{|l|}{ Fatores de risco do escore TIMI } \\
\hline Diabetes mellitus & $45(43,7)$ & 26,7 \\
\hline Hipertensão Arterial Sistêmica & $63(61,2)$ & 19,0 \\
\hline Angina & $18(17,5)$ & 22,2 \\
\hline \multicolumn{3}{|l|}{ Apresentação Clínica } \\
\hline $\mathrm{PAS}^{1}<100 \mathrm{mmHg}$ & $25(24,3)$ & 40,0 \\
\hline $\mathrm{FC}^{2}>100 \mathrm{bpm}$ & $16(15,5)$ & 50,0 \\
\hline Parada cardiorrespiratória & $4(3,9)$ & 75 \\
\hline Killip I & $72(69,9)$ & 1,4 \\
\hline Killip II - IV & $31(30,1)$ & 54,8 \\
\hline$\Delta \mathrm{T}^{3} \leq 4$ horas & $51(49,5)$ & 9,8 \\
\hline$\Delta \mathrm{T}>4$ horas & $52(50,5)$ & 25,0 \\
\hline $\mathrm{IAM}^{4}$ de parede anterior & $51(49,5)$ & 21,6 \\
\hline IAM de parede anterior extensa & $6(5,8)$ & 33,3 \\
\hline IAM de parede inferior & $53(51,5)$ & 17,0 \\
\hline
\end{tabular}

${ }^{1}$ Pressão arterial sistólica; ${ }^{2}$ frequência cardíaca; ${ }^{3}$ Tempo entre dor e primeiro atendimento; ${ }^{4}$ Infarto agudo do miocárdio.

Tabela 2 - Mortalidade intra-hospitalar pós-infarto agudo do miocárdio segundo grupos de risco pelo escore TIMI. Tubarão-SC, 2004 a 2005

\begin{tabular}{lccr}
\hline Grupo de Risco & Pontuação no escore TIMI & n ${ }^{\circ}$ de casos & Mortalidade (\%) \\
\hline Baixo Risco & 0 a 2 & 39 & 0 \\
\hline Médio Risco & 3 a 5 & 37 & 8,1 \\
\hline Alto Risco & Mais de 5 & 27 & 55,6 \\
\hline
\end{tabular}


com 75 anos ou mais, apenas 2,9\% foram tratados com estreptoquinase. Empregou-se o uso de fibrinolítico em $45,7 \%$ dos casos do sexo feminino e em $64,7 \%$ dos casos do sexo masculino. O uso de fibrinolítico foi quase $50 \%$ menor no grupo de alto risco em comparação ao de baixo risco $(R R=0,48$; IC95\%:0,27-0,85; $p=0,004)$.

Os valores máximos alcançados pelos marcadores cardíacos relacionaram-se à mortalidade hospitalar. $\mathrm{O}$ pico de creatinofosfoquinase (CK) foi inferior a dez vezes o valor normal em 52,9\% dos casos, com mortalidade hospitalar de $6,5 \%$. Dentre os $32,2 \%$ de pacientes com valores intermediários de CK (pico de CK de 10 a 20 vezes o valor normal), 14,3\% foram a óbito. A mortalidade no grupo com pico de CK superior a 20 vezes o valor normal foi de 30,8\%. Quanto à isoforma cardíaca CKMB, 33,3\% dos pacientes apresentaram pico inferior a cinco vezes o valor normal, com mortalidade de 6,9\%. Já no grupo que atingiu pico de CKMB igual ou superior a cinco vezes o valor normal, 18,2\% dos casos foram a óbito.

A mortalidade no sexo feminino foi mais que o triplo quando comparada ao masculino. Além disso, o sexo feminino esteve associado a um risco 2,1 vezes maior de pertencer ao grupo de alto risco (IC95\%: 1,23 - 3,75; $p=0,008$ ). O risco de apresentar Killip de II a IV à admissão também foi 2,1 vezes maior no sexo feminino (IC95\% $=1,17-3,68 ; p=$ $0,013)$. Do total de mulheres, $25,7 \%$ apresentaram idade de 75 anos ou mais, enquanto apenas 7,4\% dos homens tinham essa idade (Tabela 3).

Entre os pacientes Killip IV à admissão, 100\% pertenceram ao grupo de alto risco pelo escore TIMI. Além disso, 70\% dos pacientes que evoluíram para choque cardiogênico durante a evolução foram enquadrados na categoria de alto risco (Tabela 4). Dos pacientes de alto risco que ficaram 48 horas ou menos internados, 8,3\% foram transferidos e o restante foi a óbito.

A parada cardiorrespiratória foi associada a 66,7\% de mortalidade, sendo $58,3 \%$ quando o ritmo de parada foi fibrilação ventricular e 75\% quando assistolia. Pacientes com choque cardiogênico foram a óbito em $90 \%$ dos casos e não houve nenhum caso de taquicardia ventricular sustentada (Tabela 5).
O tempo médio de hospitalização foi de 9,1 \pm 8,8 dias. Pacientes com baixo risco permaneceram em média 7,9 \pm 3,0 dias internados. Para os pacientes com médio risco esse tempo foi de 10,7 \pm 9,6 dias, enquanto para os de alto risco foi de $8,5 \pm 12,4$ dias.

\section{Discussão}

Na presente amostra, a distribuição por sexo e faixas etárias foi análoga a outros estudos-6,9,14. A média de tempo de 6,7 horas entre o início dos sintomas e a primeira avaliação na sala de emergência foi inferior à descrita por Zornoff e cols. ${ }^{14}$ (10,6 horas). Por se tratar de um hospital referência na região, houve discrepância significativa no tempo entre o início dos sintomas e o atendimento médico conforme a procedência. Para os casos residentes em Tubarão, o tempo médio entre dor e atendimento foi de 4,5 horas, já nos pacientes de municípios vizinhos, foi de 8,2 horas. Outro dado que chama atenção é que $70,4 \%$ dos pacientes de alto risco aguardaram mais de quatro horas até o atendimento inicial.

A prevalência de hipertensão $(61,2 \%)$ foi próxima a de estudos brasileiros ${ }^{4-6,15}$ e superior a de estudos internacionais $\left(21,6 \%{ }^{9}\right.$ e $\left.54 \%{ }^{10}\right)$.

Dois trabalhos recentes relataram a prevalência de angina antecedendo o infarto de $46 \%{ }^{6}$ e $65 \%{ }^{16}$ dos pacientes, cifras bastante altas se comparadas aos $17,5 \%$ encontrados em nossa amostra. Outros estudos internacionais relatam $11,5 \%{ }^{10} \mathrm{e}$ $21,6 \%{ }^{9}$ de pacientes com angina antecedendo o infarto.

O estado hemodinâmico dos pacientes à admissão foi mais grave em comparação ao mencionado na literatura. Nessa amostra, a frequência de Killip II-IV foi de $30,1 \%$, superior aos $25,1 \%{ }^{10}, 17,3 \%{ }^{16}$ e $12,2 \%{ }^{9}$ encontrados em outros estudos. A pressão arterial sistólica menor que 100 mmHg à admissão esteve presente em $24,3 \%$ dos casos. Em outros estudos, essa frequência foi de $2,5 \%{ }^{9}$ a $8,7 \%{ }^{10}$.

Refutando a literatura ${ }^{4}$, neste estudo a parede mais acometida por IAM foi a inferior, ao invés da anterior. Entretanto, no grupo classificado como alto risco essa parede foi comprometida em 25,9\% dos casos.

Independentemente da técnica utilizada, a terapia de reperfusão miocárdica consiste na intervenção mais

Tabela 3 - Variáveis associadas à mortalidade hospitalar por infarto agudo do miocárdio. Tubarão-SC, 2004 a 2005

\begin{tabular}{lcc}
\hline Variável & RR & IC 95\% \\
\hline Idade igual ou superior a 75 anos & 7,9 & 3,8 a 16,6 \\
\hline Sexo feminino & 3,1 & 1,3 a 7,2 \\
\hline Diabetes mellitus & 3,7 & 1,3 a 10,8 \\
\hline Killip II -IV à admissão & 2,2 & 1,5 a 3,2 \\
\hline frequência cardíaca maior que 100 bpm & 4,3 & 2,0 a 9,0 \\
\hline Pressão arterial sistólica menor que 100 mmHg & 3,9 & 1,7 a 8,8 \\
\hline Não uso de trombolítico & 2,8 & 1,2 a 6,9 \\
\hline Presença de uma ou mais complicações hospitalares* & 4,0000 \\
\hline
\end{tabular}

* Killip II/III na evolução, dor precordial recorrente, fibrilação ventricular, assistolia, bloqueio atrioventricular de alto grau e choque cardiogênico. 


\section{Artigo Original}

Tabela 4 - Características dos pacientes com infarto agudo do miocárdio na primeira avaliação e na evolução, conforme grupos de risco. Tubarão-SC, 2004 a 2005

\begin{tabular}{lccc}
\hline Variável & Baixo Risco (\%) & Médio Risco (\%) & Alto Risco (\%) \\
\hline História médica pregressa & & & 73 \\
\hline Hipertensão arterial sistêmica & 46,2 & 24,3 & 66,7 \\
\hline Angina & 12,8 & 56,8 & 54,8 \\
\hline Diabetes mellitus & 20,5 & & 59,3 \\
\hline Apresentação clínica & & 25,8 & 74,2 \\
\hline Killip II - IV & 0 & 8,1 & 48,1 \\
\hline PAS ${ }^{1}<100$ mmHg & 0 & 13,5 & 51,9 \\
\hline FC ${ }^{2}>100$ bpm & 15,4 & 56,8 & 70,4 \\
\hline$\Delta T^{3}>4$ horas & 38,2 & 16,2 & 25,9 \\
\hline IAM ${ }^{4}$ de parede anterior extensa & 10,3 & 64,9 & 33,3 \\
\hline Tratamento com trombolítico & 69,2 & 5,4 & 44,4 \\
\hline Tempo de internação $\leq 48 h$ & 0 & 13,5 & 11,1 \\
\hline Transferência para outro hospital & 17,9 & 62,2 & 70,4 \\
\hline Presença de uma ou mais complicações hospitalares ${ }^{5}$ & 30,8 & & \\
\hline
\end{tabular}

Tabela 5 - Mortalidade relacionada às complicações hospitalares pós-infarto agudo do miocárdio. Tubarão-SC, 2004 a 2005

\begin{tabular}{lcc}
\hline Complicação & Frequência (\%) & Mortalidade (\%) \\
\hline Killip I//III & 32 & 27,3 \\
\hline Dor precordial recorrente & 22,3 & 13 \\
\hline Fibrilação ventricular & 11,7 & 58,3 \\
\hline Bloqueio atrioventricular de alto grau & 10,7 & 45,5 \\
\hline Choque cardiogênico & 9,7 & 90 \\
\hline Assistolia & 7,8 & 75 \\
\hline
\end{tabular}

importante no manejo de pacientes infartados ${ }^{3}$. Um estudo nacional estimou que o percentual de indicação de trombólise no Brasil é de $40 \%{ }^{17}$. Estudos nacionais e internacionais relataram $36,8 \%{ }^{17}, 39 \%{ }^{14}, 48 \%{ }^{10}$ e $35,1 \%^{4}$ de uso de terapia de reperfusão. Neste estudo, 58,3\% dos pacientes usaram estreptoquinase, entretanto, no grupo estratificado como alto risco, lançou-se mão dessa terapia em apenas 33,3\% dos casos. Um estudo internacional também destaca que pacientes não submetidos à terapia de reperfusão apresentam maior frequência de características de alto risco e escores TIMI mais altos ${ }^{10}$.

O tempo médio de internação foi semelhante ao relatado em outro estudo (9 dias) ${ }^{6}$. Nenhum paciente de baixo risco permaneceu menos de 48 horas no hospital, entretanto, $44,4 \%$ dos pacientes de alto risco receberam alta nesse período. Aproximadamente $67 \%$ dos pacientes que ficaram menos de 48 horas internados foram a óbito.
No estudo In $\mathrm{TIMI}^{9}, 50 \%$ dos pacientes se enquadraram como baixo risco e apenas $12 \%$ como alto risco ${ }^{9}$. Neste trabalho, 26,2\% dos casos eram de alto risco e $37,9 \%$ baixo risco. Nessa amostra, dor precordial recorrente, fibrilação ventricular, bloqueio atrioventricular de alto grau e choque cardiogênico ocorreram em frequência semelhante à descrita por outros autores $\mathrm{s}^{3,4,14,15}$.

A mortalidade hospitalar em pacientes não selecionados com IAM tem variado entre $10 \%$ e $20 \%{ }^{18}$. A mortalidade de $17,5 \%$ encontrada na presente amostra é semelhante à de outros trabalhos brasileiros ${ }^{4-6,19} \mathrm{e}$ internacionais $(12,6 \%)^{10}$. Esses valores se distanciam dos 6,1\% encontrados em um ensaio clínico randomizado como o In TIMI ${ }^{9}$.

A identificação de variáveis preditoras de maior mortalidade hospitalar pós-IAM têm sido descrita por muitos autores ${ }^{4,6,14,19}$. Vários estudos têm proposto escores prognósticos baseados 
nas variáveis com maior impacto na mortalidade em análise multivariada. Nesse sentido, o escore TIMI manteve uma forte associação com a mortalidade, sendo que o risco de morte para pacientes com escore maior ou igual a oito foi 40 vezes maior em relação aos com escore zero ${ }^{9}$. Além disso, escores maiores que cinco identificaram no estudo In $\mathrm{TIMI}^{9} 12 \%$ dos pacientes com risco de mortalidade maior que o dobro da média da população.

Bassam e cols. ${ }^{19}$ consideraram baixo risco grupos com mortalidade abaixo de $10 \%$, e alto risco grupos com mortalidade acima de $40 \%{ }^{19}$. Ketzer e cols. ${ }^{13}$, para analisar a mortalidade por infarto seis meses após o evento agudo, dividiram os pacientes em grupos de risco conforme a pontuação no escore $\mathrm{TIMI}^{13}$. Os resultados encontrados foram: $4,1 \%$ de mortalidade no grupo de baixo risco, $10 \%$ no de médio risco e $52 \%$ no de alto risco. No presente estudo, a mortalidade foi de 55,6\% no grupo de alto risco, $8,1 \%$ no de médio risco e $0 \%$ no de baixo risco.

A mortalidade pós-IAM aumenta dramaticamente com a idade, subindo de 2,1\% em pacientes com menos de 55 anos para $26,3 \%$ em pacientes com 85 anos ou mais ${ }^{20}$. Idosos possuem reserva coronária e miocárdica reduzidas ${ }^{19,21}$, uma vez que têm maior incidência de diabete, hipertensão, infarto prévio e insuficiência cardíaca ${ }^{22}$. Neste estudo, a mortalidade aumentou de 8,1\% em pacientes com menos de 65 para $71,4 \%$ em pacientes com 75 anos ou mais. Parte desse aumento pode ser atribuído ao perfil de risco mais grave dos idosos. Nessa amostra, 48,1\% dos pacientes do grupo de alto risco tinham mais de 75 anos, contra nenhum caso acima de 64 anos no grupo de baixo risco. Não houve diferenças significativas quanto ao tempo decorrido entre o início dos sintomas e o atendimento médico pela idade.

Em análise multivariada, a classe de Killip-Kimball é o preditor mais poderoso de prognóstico, com aumento de duas vezes no risco de morte a cada piora de classe ${ }^{16}$. Outros autores também destacam a classe de Killip como a variável independente mais relacionada à mortalidade ${ }^{4,9,19,22}$. Neste estudo, 54,8\% dos pacientes com Killip de II a IV foram a óbito.

Embora não haja unanimidade na literatura atribuindo o sexo feminino como variável independente associada à mortalidade por IAM, vários autores ${ }^{4,22-24}$ verificaram essa associação. Esse fato está ligado à severidade intrínseca da doença coronariana em mulheres, à faixa etária maior acometida, ao maior número de comorbidades ${ }^{22}$ e à subutilização de intervenções terapêuticas eficazes ${ }^{25}$.

Nessa amostra, o sexo feminino conferiu um risco de óbito 3,1 vezes maior. Parte da maior mortalidade no sexo feminino pode ser atribuída à idade mais elevada. Entretanto, o perfil de risco mais grave dessas pacientes também contribui para alta taxa de óbito. Neste estudo, o risco de apresentar Killip não I à admissão e pertencer à categoria de alto risco foi maior nas mulheres. Vaccarino e cols. ${ }^{26}$ chamam atenção para a maior probabilidade de mulheres apresentarem quadro clínico mais severo, no entanto, ressaltam que isso é válido apenas para mulheres jovens ${ }^{26}$. Não houve diferença significativa no tempo médio entre dor e atendimento para ambos os sexos.
O diabete manteve-se como uma variável independente associada à mortalidade em análises multivariadas de vários estudos ${ }^{4,9,16,22}$. Ribeiro e cols. ${ }^{22}$ descreveram uma chance de óbito 2,3 vezes maior em diabéticos ${ }^{22}$. O risco de óbito foi 3,7 vezes maior em diabéticos neste estudo. Além disso, na amostra em tela, o grupo de alto risco apresentou uma frequência maior de diabete $(60 \%)$ em comparação ao de baixo risco (20\%)

Em conformidade ao encontrado na literatura ${ }^{3}$, houve associação entre o tempo decorrido do início dos sintomas e o primeiro atendimento à maior mortalidade. Pacientes que levaram mais que quatro horas para procurar assistência médica, tiveram mortalidade de $25 \%$. Aqueles que demoraram quatro horas ou menos, apresentaram 9,8\% de óbito. História prévia de hipertensão e angina também foram observadas, aumentando o risco de morte pós-infarto no presente estudo ${ }^{9,10}$.

No estudo GRACE ${ }^{16}$, para cada aumento de 30 batimentos na frequência cardíaca o risco de morte aumentou em 20\%. Da mesma forma, a redução $20 \mathrm{mmHg}$ na pressão arterial sistólica aumentou em 1,3 vezes a mortalidade naquele estudo. No estudo $\ln \mathrm{TIMI}^{9}$, a pressão arterial sistólica menor que $100 \mathrm{mmHg}$ conferiu uma chance 2,7 vezes maior de morte. No mesmo estudo, a frequência cardíaca maior que $100 \mathrm{bpm}$ foi associada a um risco de óbito 2,3 vezes maior. Em nossa amostra, pacientes com hipotensão e taquicardia também tenderam a maior mortalidade.

Ribeiro e cols..$^{22}$ relatam mortalidade de $16,7 \%$ no grupo de pacientes em que se usou trombolítico e de $28,7 \%$ no grupo em que não se lançou mão dessa terapia. No presente estudo, o não uso de estreptoquinase conferiu risco de óbito 2,8 vezes maior. Grupos com maior risco de morte são menos submetidos à terapia de reperfusão $0^{3,10,16}$. Nessa amostra, o risco de receber fibrinolítico foi $50 \%$ menor entre os casos de alto risco. Um estudo recente mostra que 46,2\% dos casos abaixo de 55 anos são tratados com trombolítico, enquanto apenas 9,5\% daqueles com 85 anos ou mais recebem o mesmo tratamento ${ }^{19}$. Em nossa população, apenas 2,9\% dos casos acima de 75 anos receberam fibrinolítico. A prevalência de uso de estreptoquinase foi menor nas mulheres $(45,7 \%)$ quando comparadas aos homens $(64,7 \%)$.

A presença de complicações hospitalares pós-infarto confere um pior prognóstico aos pacientes ${ }^{3,4,8}$. Nessa população, o risco de morte para os casos com uma ou mais complicações pós-infarto foi 4,5 vezes maior. Escores TIMI mais altos estiveram associados, como esperado, a frequências maiores de complicações. Entre os casos que evoluíram com choque cardiogênico, $70 \%$ foram caracterizados como alto risco na admissão, e 90\% dos pacientes com choque cardiogênico foram a óbito.

\section{Conclusões}

Na amostra estudada, oito variáveis - idade acima de 75 anos; diabete; classe Killip; frequência cardíaca acima de 100 bpm; pressão arterial sistólica abaixo de 100 mmHg; não uso de trombolíticos; e complicações - estiveram 


\section{Artigo Original}

significativamente associadas ao maior risco de óbito hospitalar pós-infarto, sendo as maiores: idade superior a 75 anos, presença de complicações e frequência cardíaca acima de 100 bpm.

\section{Potencial Conflito de Interesses}

Declaro não haver conflito de interesses pertinentes.

\section{Fontes de Financiamento}

O presente estudo não teve fontes de financiamento externas.

\section{Vinculação Acadêmica}

Este artigo é parte de tese de conclusão de curso Jaqueline Locks Pereira pela Universidade do Sul de Santa Catarina.

\section{Referências}

1. Timerman A, Santos RD, Sousa MFM, Serrano Jr CV. Aspectos epidemiológicos das doenças cardiovasculares em nosso meio: tendência da mortalidade por doença isquêmica do coração no Brasil de 1979 a 1996. Rev Soc Cardiol Estado de São Paulo. 2002; 11 (4): 715-23.

2. Hom T, Haase N, Rosamend W, Howard VJ, Rumsfeld J, Manolio T, et al. Heart disease and stroke statistics--2006 update: a report from the American Heart Association Statistics Committee and Stroke Statistics Subcommittee. Circulation. 2006; 113 (6): 85-151.

3. Sociedade Brasileira de Cardiologia. III Diretriz sobre tratamento do infarto agudo do miocárdio. Arq Bras Cardiol. 2004; 83 (4): 8-86.

4. Escosteguy CC, Portela MC, Medronho RA, Vasconcellos MTL. Infarto agudo do miocárdio: perfil clínico-epidemiológico e fatores associados ao óbito hospitalar no município do Rio de Janeiro. Arq Bras Cardiol. 2003; 80 (6): 593-9.

5. Mattos MA, Toledo DG, Mattos CE, Tura BR, Gadelha DNB, Siqueira Fo AG Tendência temporal de letalidade hospitalar por infarto agudo do miocárdio: 1994-2003. Arq Bras Cardiol. 2005; 84 (5): 416-21.

6. Berwnger $\mathrm{O}$, Avezum A, Guimarães HP. Epidemiologia da síndrome isquêmica aguda com supradesnivelamento do segmento ST- ênfase nas características brasileiras. Rev Soc Cardiol Estado de São Paulo. 2004; 6: 833-9.

7. Peterson ED, Shaw LJ, Califf RM. Clinical Guideline (Part II): Risk stratification after myocardial infarction. Ann Intern Med. 1997; 126: 561-82.

8. Mark DB, Sigmon K, Topol EJ, Kereiakes DJ, Pryos DB, Candela RJ, et al. Identification of acute myocardial infarction patients suitable for early hospital discharge after aggressive interventional therapy: results from the thrombolysis and angioplasty in acute myocardial infarction Registry. Circulation. 1991; 83: 1186-93.

9. Morrow DA, Antman EM, Charlesworth A, Cairns R, Murphy SA, Lemos JA, et al. TIMI risk score for ST-elevation Myocardial Infarction: a convenient, bedside, clinical score for risk assessment at presentation: an Intravenous nPA for Treatment of Infarcting Myocardium Early II Trial Substudy. Circulation. 2000; 102: 2031-7.

10. Morrow DA, Antman EM, Parsons L, Lemos JA, Cannon CP, Giugliano RP, et al. Application of the TIMI risk score for ST-elevation MI in the National Registry of Myocardial Infarction 3. JAMA. 2001; 286 (11): 1356-9.

11. Singh M, Reeder GS, Jacobsen SJ, Weston S, Killian J, Roger VL. Scores for post-myocardial infarction risk stratification in the community. Circulation. 2002; 106: 2309-14.

12. Marx BF, Feinstein AR. Methodologic sources of inconsistent prognoses for post-acute myocardial infarction. Am J Med. 1995; 98 (6): 537-50.

13. Ketzer BM, Gil E, Rodrigues GR, Kawata MF, Gun C, De Luca FA. Análise do TIMI Risk Score em pacientes com infarto agudo do miocárdio com supradesnivelamento do segmento ST após 6 meses. Rev Med (São Paulo). 2003; 82 (1-4): 58-66
14. Zornoff LAM, Paiva SA, Assalin VM, Pola PM, Becker LE, Okoshi MP, et al. Perfil clínico, preditores de mortalidade e tratamento de pacientes após infarto agudo do miocárdio, em hospital terciário universitário. Arq Bras Cardiol. 2002; 78 (4): 396-405.

15. Passos LC, Lopes AA, de Souza CA, Souza JA, de Souza Neto JP, Souza Fo J, et al. Preditores da mortalidade hospitalar na era trombolítica para o infarto agudo do miocárdio em Salvador (BA). Arq Bras Cardiol. 1997; 68 (4): 249-55.

16. Granger CB, Goldberg RJ, Dabbous O, Pieper KS, Eagle KA, Cannon CP, et al. Predictors of hospital mortality in the Global Registry of Acute Coronary Events. Global Registry of Acute Coronary Events Investigators. Arch Intern Med. 2003; 163 (19): 2345-53.

17. Krauss Silva L, Escosteguy CC, Machado CV. Metodologia para a estimativa de padrões de qualidade: o caso do infarto agudo do miocárdio. Cad Saúde Pública. 1996; 125 (supl. 2): 71-83.

18. Gunnar RM, Bourdillon PD, Dixon DW, Fuster V, Karp RB, Kennedy JW, et al. ACC/AHA guidelines for the early management of patients with acute myocardial infarction: a report of the ACC/AHA Task Force on Assessment of Diagnostic and Therapeutic Cardiovascular Procedures. Circulation. 1990; 82 (2): 664-707.

19. Bassan R, Potsch A, Pimenta L, Tachibana V, Souza A, Migon H, et al. Mortalidade hospitalar no infarto agudo do miocárdio: é possível prever utilizando dados de admissão?. Arq Bras Cardiol. 1996; 67 (3): 149-58.

20. Boucher JM, Racine N, Thanh TH, Rahma E, Brophy J, Le Lorier J, et al. Age - related differences in in-hospital mortality and the use of thrombolytic therapy for acute myocardial infarction. CMAJ. 2001; 164 (9): 1285-90.

21. Leal MF, Stadler NF, Souza Filho NFS, Haggi Filho H, Klsoviski ER, Munhoz EC. Acute myocardial infarction in elderly patients: comparative analysis of the predictors of mortality: the elderly versus young. Arq Bras Cardiol. 2002; 79 (4): 369-74.

22. Ribeiro DGL, Andrade PJN, Paes Júnior NJ, Saraiva LR. Infarto agudo do miocárdio: preditores de mortalidade em hospital público em Fortaleza, Ceará. Arq Bras Cardiol. 2003; 80 (6): 607-13.

23. Pimenta L, Bassam R, Potsch A, Soares JF, Albanesi Filho FM. É o sexo feminino um preditor independente de mortalidade hospitalar no infarto agudo do miocárdio? Arq Bras Cardiol. 2001; 77: 37-43.

24. Greenland P, Reicher-Reiss H, Goldbourt U, Behar S. In-hospital and 1-year mortality in 1524 women after myocardial infarction: comparison women with 4315 men. Circulation. 1991; 83 (2): 484-91.

25. Passos LCS, Lopes AA, Lessa I, Sanches A, Santos JR. Tendência da mortalidade por infarto agudo do miocárdio (1981 a 1996) na cidade de Salvador, Brasil. Arq Bras Cardiol. 2000; 74: 329-31.

26. Vaccarino V, Parsons L, Every NR, Barron HV, Krumholz HM. Sex-based differences in early mortality after myocardial infarction. National Registry of Myocardial Infarction 2 Participants. N Engl J Med. 1999; 341 (4): 217-25. 
Artigo Original

Anexo 1 - Escore de risco TIMI $^{9}$

\begin{tabular}{lc}
\hline Histórico & Pontos \\
\hline Idade $\geq 75$ anos & 3 \\
\hline $65-74$ anos & 2 \\
\hline DM ou HAS ou Angina & 1 \\
\hline Exame Clínico & \\
\hline PAS $<100$ mmHg & 3 \\
\hline FC $>100$ bpm & 2 \\
\hline Killinp II-IV & 2 \\
\hline Peso $<67$ Kg & 1 \\
\hline Apresentação clínica & \\
\hline $\begin{array}{l}\text { Elevação de ST na parede } \\
\text { anterior ou bloqueio de ramo } \\
\text { esquerdo }\end{array}$ & 1 \\
\hline Tempo de apresentação $>4 h$ & 1 \\
\hline
\end{tabular}

\begin{tabular}{lc}
\hline Escore de risco & Mortalidade Intra-hospitalar (\%) \\
\hline 0 & 0,7 \\
\hline 1 & 0,3 \\
\hline 2 & 1,9 \\
\hline 3 & 3,9 \\
\hline 4 & 6,5 \\
\hline 5 & 11,6 \\
\hline 6 & 14,7 \\
\hline 7 & 21,5 \\
\hline 8 & 24,4 \\
\hline$>8$ & 31,7 \\
\hline
\end{tabular}

